\title{
Formation of a disk structure in the symbiotic binary AX Persei during its 2007-10 precursor-type activity ${ }^{\star}$
}

\author{
A. Skopal ${ }^{1, \star \star}$, T. N. Tarasova ${ }^{2}$, Z. Cariková ${ }^{1}$, F. Castellani ${ }^{3}$, G. Cherini ${ }^{3}$, S. Dallaporta ${ }^{3}$, A. Frigo ${ }^{3}$, C. Marangoni ${ }^{3}$, \\ S. Moretti ${ }^{3}$, U. Munari ${ }^{4}$, G. L. Righetti ${ }^{3}$, A. Siviero ${ }^{5}$, S. Tomaselli $^{3}$, A. Vagnozzi ${ }^{3}$, and P. Valisa ${ }^{3}$
}

\author{
1 Astronomical Institute, Slovak Academy of Sciences, 05960 Tatranská Lomnica, Slovakia \\ 2 Crimean Astrophysical Observatory, Nauchny, Ukraine \\ 3 ANS Collaboration, c/o Osservatorio Astronomico di Padova, Sede di Asiago, 36032 Asiago (VI), Italy \\ 4 INAF Osservatorio Astronomico di Padova, Sede di Asiago, 36032 Asiago (VI), Italy \\ 5 Dipartimento di Astronomia, Universita' di Padova, Osservatorio Astrofisico, 36012 Asiago (VI), Italy
}

Received 28 March 2011 / Accepted 12 September 2011

\section{ABSTRACT}

\begin{abstract}
Context. AX Per is an eclipsing symbiotic binary. During active phases, deep narrow minima are observed in its light curve, and the ionization structure in the binary changes significantly. From 2007.5, AX Per entered a new active phase.

Aims. We aim to derive the ionization structure in the binary and its changes during the recent active phase.

Methods. We used optical high- and low-resolution spectroscopy and $U B V R_{\mathrm{C}} I_{\mathrm{C}}$ photometry. We modeled the SED in the optical and broad wings of the $\mathrm{H} \alpha$ line profile during the 2007-10 higher level of the AX Per activity.

Results. After 10 orbital cycles ( 18.6 years), we again measured the eclipse of the hot component by its giant companion in the light curve. We derived a radius of $27 \pm 2 R_{\odot}$ for the eclipsed object and $115 \pm 2 R_{\odot}$ for the eclipsing cool giant. The new active phase was connected with a significant enhancement of the hot star wind. From quiescence to activity, the mass-loss rate increased from $\sim 9 \times 10^{-8}$ to $\sim 3 \times 10^{-6} M_{\odot} \mathrm{yr}^{-1}$, respectively. The wind causes the emission of the $\mathrm{He}^{++}$zone, located in the vicinity of the hot star, and also is the reason for the fraction of the [O III] zone at farther distances. Simultaneously, we identified a variable optically thick warm $\left(T_{\text {eff }} \sim 6000 \mathrm{~K}\right)$ source that contributes markedly to the composite spectrum. The source was located at the hot star's equator and has the form of a flared disk, whose outer rim simulates the warm photosphere.

Conclusions. The formation of the neutral disk-like zone around the accretor during the active phase was connected with its enhanced wind. It is probable that this connection represents a common origin of the warm pseudophotospheres that are indicated during the active phases of symbiotic stars.
\end{abstract}

Key words. stars: fundamental parameters - stars: winds, outflows - binaries: symbiotic - stars: individual: AX Per

\section{Introduction}

Symbiotic stars are interacting binary systems comprising a cool giant and a hot compact star, mostly a white dwarf (WD), on, typically, a few years' orbit. The WD accretes from the giant's wind, heats up to $1-2 \times 10^{5} \mathrm{~K}$, and becomes as luminous as $10^{2}-10^{4} L_{\odot}$. It ionizes the circumbinary environment and causes nebular emission. As a result, the spectrum of symbiotic stars consists of three basic components of radiation - two stellar and one nebular. If the processes of the mass loss, accretion and ionization are in a mutual equilibrium, the symbiotic system releases its energy approximately at a constant rate and spectral energy distribution (SED). This stage is called the quiescent phase. Once this equilibrium is disturbed, the symbiotic system changes its radiation significantly, brightens in the optical by a few magnitudes and usually shows signatures of a mass outflow for a few months to years. We name this stage the active phase. Many particular aspects of this general view have been originally pointed out by, e.g., Friedjung \& Viotti (1982), Seaquist et al. (1984), Kenyon \& Webbink (1984), Nussbaumer \& Vogel (1987), and are more recently discussed in Corradi et al. (2003).

\footnotetext{
$\star$ Tables 2, 3 and 5 are available at the CDS via anonymous ftp to cdsarc.u-strasbg.fr $(130.79 .128 .5)$ or via

http://cdsarc.u-strasbg.fr/viz-bin/qcat?]/A+A/536/A27

$\star \star$ Visiting Astronomer: Asiago Astrophysical Observatory.
}

To explain the high luminosity of hot components in symbiotic binaries, it was suggested that they are powered by stable hydrogen nuclear burning on the WD surface, which requires a certain range of accretion rates (e.g. Paczyński \& Żytkow 1978; Fujimoto 1982). During active phases, we observe a significant cooling of the spectrum produced by the hot star (e.g. Kenyon \& Webbink 1984, and references therein). The event of outbursts could result from an increase in the accretion rate above that which sustains the stable burning, which leads to an expansion of the burning envelope to an A-F type (pseudo)photosphere (e.g. Tutukov \& Yangelson 1976; Paczyński \& Rudak 1980). As a result, the pseudophotosphere will radiate at a lower temperature, and consequently shift the maximum of its SED from shorter to longer wavelengths, causing a brightening in the optical. Based on optical observations, this scenario was supported by many authors. Recently, Siviero et al. (2009) applied it to the 200809 active phase of CI Cyg. However, modeling the UV/near-IR continuum of symbiotic binaries with a high orbital inclination during active phases indicated an increase of both the stellar radiation from a warm $\left(1-2 \times 10^{4} \mathrm{~K}\right)$ pseudophotosphere and the nebular radiation within the optical wavelengths. This led to the suggestion that there is an edge-on disk around the accretor, whose outer flared rim represents the warm pseudophotosphere, and the nebula is placed above/below the disk (Skopal 2005). In 

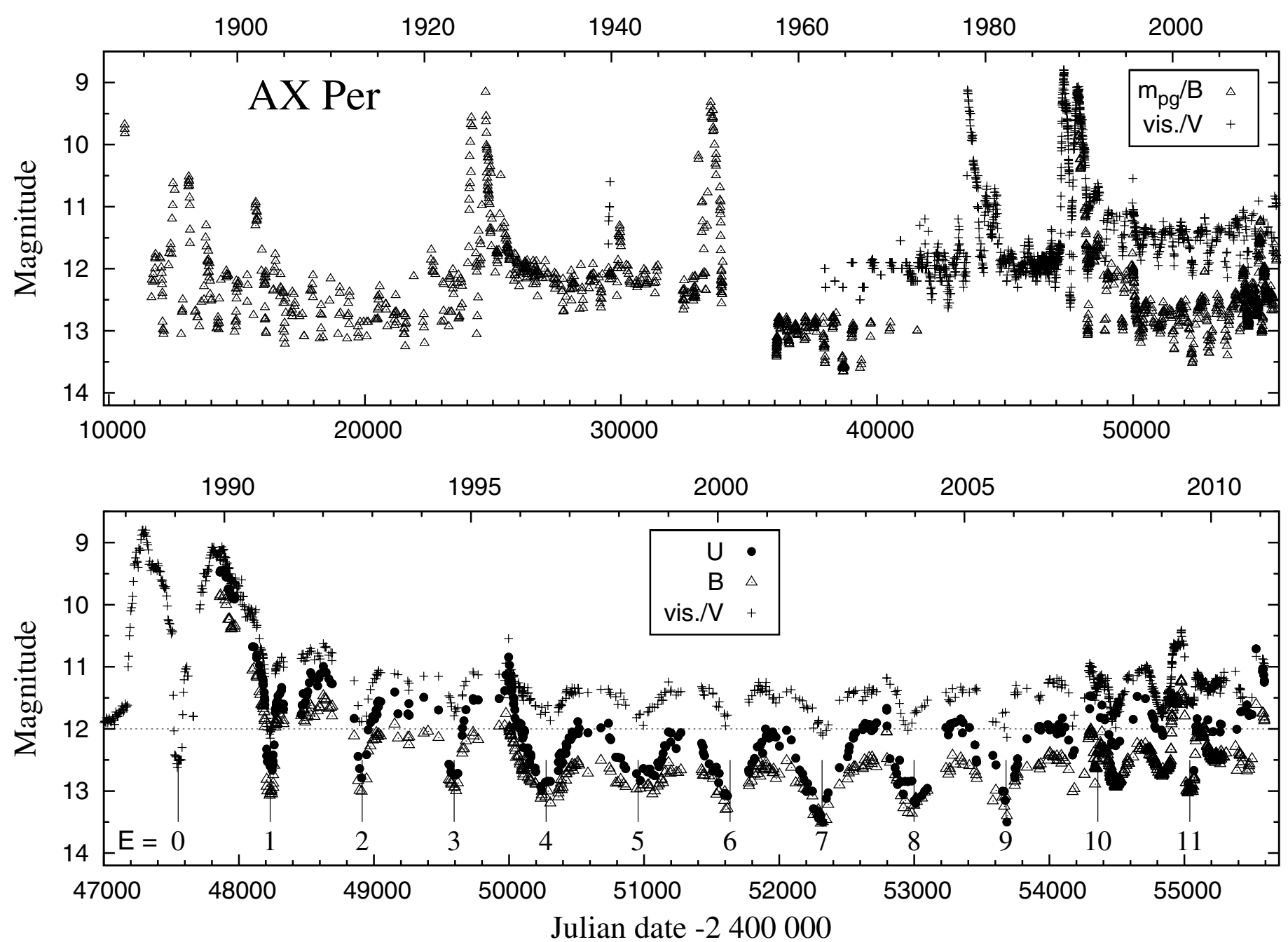

Fig. 1. Top panel displays the historical LC of AX Per from $\sim 1890$ (see Fig. 1 of Skopal et al. 2001, and references therein). We used visual magnitude estimates from the AAVSO International Database and those gathered by members of the AFOEV, which are available on CDS. Visual data were smoothed within 20-day bins. Bottom panel shows the $U B V$ LCs of AX Per from its last 1988 major outburst. Minima timing corresponds to the ephemeris given by Eq. (1).

this contribution we test this scenario on the recent active phase of the symbiotic binary AX Per.

At present, AX Per is known as an eclipsing symbiotic binary (Skopal 1991) comprising a M4.5 III giant (Mürset \& Schmid 1999) and a WD on a 680-d orbit (e.g. Mikolajewska \& Kenyon 1992; Fekel et al. 2000). The last active phase began in 1988, when AX Per brightened by $\sim 3$ mag in the visual, developed a specific phase-dependent modulation in the light curve (LC), and showed narrow eclipses at the position of the inferior conjunction of the giant (e.g. Ivison et al. 1993). Transition to quiescence happened during 1995, when the LC profile turned to the wavelike orbitally related variation at a low level of the star's brightness (Skopal et al. 2001, Fig. 1 here). Recently, Munari et al. (2009a) reported on a rapid increase in the AX Per brightness by $\sim 1$ mag in $B$ during 2009 March. They pointed a similarity between this brightening phase and the short-duration flare that occurred in the AX Per LC about one year before its major 19881995 active phase. Recently, Munari et al. (2010) reported on a rise in brightness of AX Per during 2010 November by $\sim 0.7 \mathrm{mag}$ in $B$, which supported their previous suggestion that the 200710 active phase was a precursor to an oncoming major outburst. From this point of view, understanding the precursor-type activity can aid us in understanding the nature of the $\mathrm{Z}$ And-type outbursts better.
Accordingly, in this paper we investigate the structure of the hot component in the binary (i.e. the hot star with the warm pseudophotosphere and the nebula) that developed during the 2007-10 brighter phase of AX Per. To achieve this aim, we analyze our multicolour photometric observations and the low- and high-resolution optical spectra. In Sect. 2 we summarize and describe our observations and data reduction. Section 3 describes our analysis and presents the results. Their discussion and conclusions are found in Sects. 4 and 5, respectively.

\section{Observations and data reduction}

Our observations of AX Per during its 2007-10 higher level of activity were carried out at different observatories.

Spectroscopic observations were secured with five different instruments: (1) the 1.22-m telescope, operated in Asiago by the Department of Astronomy of the University of Padova, was used with a B\&C spectrograph, $300 \mathrm{ln} / \mathrm{mm}$ grating and ANDOR iDus 440A CCD camera, equipped with an EEV 42-10BU back illuminated chip $(2048 \times 512$ pixels of $13.5 \mu \mathrm{m}$ size $)$; (2) the 1.82-m telescope, operated in Asiago by INAF Astronomical Observatory of Padova, mounting a REOSC echelle spectrograph equipped with an AIMO E2VCCD47-10 back-illuminated CCD detector $(1100 \times 1100$ pixels of $13 \mu \mathrm{m}$ size $)$; (3) the $0.60-\mathrm{m}$ 
Table 1. Log of spectroscopic observations.

\begin{tabular}{lccccc}
\hline \hline Date & $\begin{array}{c}\text { Julian date } \\
+2450000\end{array}$ & $\begin{array}{c}\text { Res. } \\
\text { Power }\end{array}$ & $\begin{array}{c}\text { Disp. } \\
(\AA / \text { pix })\end{array}$ & $\begin{array}{c}\lambda \text { range } \\
(\AA)\end{array}$ & Telescope \\
\hline $2007 / 07 / 31$ & 4312.876 & 10000 & & $\mathrm{H} \beta, \mathrm{H} \alpha$ & DDO $1.88 \mathrm{~m}$ \\
$2007 / 11 / 20$ & 4425.529 & & 1.81 & $3824-7575$ & CrAO $2.6 \mathrm{~m}$ \\
$2007 / 12 / 03$ & 4438.438 & & 2.30 & $3230-7770$ & Asiago $1.22 \mathrm{~m}$ \\
$2008 / 01 / 23$ & 4488.506 & 10000 & & $\mathrm{H} \beta, \mathrm{H} \alpha$ & DDO $1.88 \mathrm{~m}$ \\
$2008 / 07 / 07$ & 4655.473 & & 1.81 & $3774-7574$ & CrAO 2.6m \\
$2008 / 08 / 10$ & 4689.450 & & 1.81 & $3749-7575$ & CrAO $2.6 \mathrm{~m}$ \\
$2008 / 09 / 24$ & 4734.609 & & 1.81 & $3749-7575$ & CrAO $2.6 \mathrm{~m}$ \\
$2008 / 10 / 13$ & 4753.638 & 20000 & & $3690-7300$ & Asiago $1.82 \mathrm{~m}$ \\
$2008 / 10 / 23$ & 4763.417 & & 1.81 & $3349-7149$ & CrAO $2.6 \mathrm{~m}$ \\
$2008 / 11 / 08$ & 4778.393 & & 1.81 & $3749-7500$ & CrAO $2.6 \mathrm{~m}$ \\
$2008 / 12 / 08$ & 4809.390 & & 2.30 & $3250-7830$ & Asiago $1.22 \mathrm{~m}$ \\
$2008 / 12 / 21$ & 4822.437 & 20000 & & $3690-7300$ & Asiago $1.82 \mathrm{~m}$ \\
$2009 / 01 / 09$ & 4841.426 & 20000 & & $3690-7300$ & Asiago $1.82 \mathrm{~m}$ \\
$2009 / 03 / 24$ & 4915.422 & & 2.12 & $3890-7930$ & Varese $0.60 \mathrm{~m}$ \\
$2009 / 04 / 01$ & 4923.323 & & 2.30 & $4200-7200$ & Asiago $1.22 \mathrm{~m}$ \\
$2009 / 04 / 07$ & 4929.303 & & 2.12 & $3900-8120$ & Varese $0.60 \mathrm{~m}$ \\
$2009 / 04 / 08$ & 4930.301 & & 2.30 & $3340-7620$ & Asiago $1.22 \mathrm{~m}$ \\
$2009 / 04 / 09$ & 4931.262 & & 1.81 & $3600-7575$ & CrAO $2.6 \mathrm{~m}$ \\
$2009 / 04 / 14$ & 4936.281 & & 2.30 & $3560-7580$ & Asiago $1.22 \mathrm{~m}$ \\
$2009 / 04 / 14$ & 4936.338 & 17000 & & $4030-7510$ & Varese $0.60 \mathrm{~m}$ \\
$2009 / 05 / 05$ & 4957.458 & & 2.12 & $3850-8270$ & Varese $0.60 \mathrm{~m}$ \\
$2009 / 08 / 05$ & 5049.535 & & 2.30 & $3320-7660$ & Asiago $1.22 \mathrm{~m}$ \\
$2009 / 08 / 26$ & 5070.565 & & 1.81 & $3324-7493$ & CrAO 2.6m \\
$2009 / 09 / 24$ & 5099.584 & & 1.81 & $3350-7500$ & CrAO 2.6m \\
$2009 / 11 / 10$ & 5146.485 & 20000 & & $3690-7300$ & Asiago $1.82 \mathrm{~m}$ \\
$2010 / 08 / 07$ & 5416.368 & & 2.12 & $3910-8540$ & Varese $0.60 \mathrm{~m}$ \\
$2010 / 08 / 16$ & 5425.564 & & 1.81 & $3358-7500$ & CrAO $2.6 \mathrm{~m}$ \\
$2010 / 08 / 21$ & 5430.348 & 17000 & & $3950-8640$ & Varese $0.60 \mathrm{~m}$ \\
$2010 / 09 / 14$ & 5454.560 & & 1.81 & $3274-7574$ & CrAO $2.6 \mathrm{~m}$ \\
\hline
\end{tabular}

telescope of the Schiaparelli Observatory in Varese, mounting a multi-mode spectrograph, able to provide both single dispersion, low-resolution spectra and Echelle spectra. The detector was an SBIG ST-10XME camera $(2194 \times 51472$ pixels of $6.8 \mu \mathrm{m}$ size); (4) the $1.88-\mathrm{m}$ telescope of the David Dunlap Observatory (DDO), University of Toronto (DDO), equipped with a single-dispersion spectrograph equipped with a Jobin Yovon Horiba CCD detector $(2048 \times 512$ pixels of $13.5 \mu \mathrm{m}$ size); and finally (5) the 2.6-m Shajn telescope, operated by the Crimean Astrophysical Observatory (CrAO) in Nauchny, mounting a SPEM spectrograph in the Nasmith focus. The detector was a SPEC-10 CCD camera $(1340 \times 100$ pixel). Table 1 provides a journal of the spectroscopic observations. At each telescope the observations were carried out as multiple exposures to avoid saturation of the strongest emission lines. Various spectrophotometric standards were observed each night to flux into absolute units the spectra of AX Per. The accuracy of the flux scale was checked against the photometric observations by integration over the fluxed spectra of the $U B V R_{\mathrm{C}} I_{\mathrm{C}}$ photometric pass-bands. Correction for flat-, bias- and dark frames was carried out in a standard way with IRAF, as well subtraction of the background sky and scattered light.

Multicolour $B V R_{\mathrm{C}} I_{\mathrm{C}} \mathrm{CCD}$ photometry was obtained with various telescopes operated by the ANS (Asiago Novae and Symbiotic Stars) Collaboration. Treatments for flat-, bias- and dark frames was carried out in a standard way. Photometric calibration and correction for color equations was carried out against the photometric sequence calibrated by Henden \& Munari (2006) around AX Per. The resulting data are presented in Table 2. Their uncertainties are the overall budget errors (which
Table 4. Effect of emission lines on the $U B V R_{\mathrm{C}}$ magnitudes.

\begin{tabular}{lcccc}
\hline \hline Date & $\Delta U_{l}$ & $\Delta B_{l}$ & $\Delta V_{l}$ & $\Delta R_{\mathrm{C}, l}$ \\
\hline $2007 / 11 / 20$ & - & -0.26 & -0.07 & -0.20 \\
$2007 / 12 / 03$ & -0.14 & -0.24 & -0.05 & -0.15 \\
$2008 / 07 / 07$ & -0.10 & -0.30 & -0.06 & -0.20 \\
$2008 / 08 / 10$ & -0.11 & -0.33 & -0.07 & -0.22 \\
$2008 / 09 / 24$ & -0.12 & -0.34 & -0.07 & -0.24 \\
$2008 / 10 / 23$ & -0.20 & -0.35 & -0.08 & -0.25 \\
$2008 / 11 / 08$ & - & -0.34 & -0.07 & -0.24 \\
$2008 / 12 / 08$ & -0.18 & -0.32 & -0.06 & -0.21 \\
$2009 / 03 / 24$ & - & -0.19 & -0.05 & -0.13 \\
$2009 / 04 / 01$ & - & -0.15 & -0.06 & -0.12 \\
$2009 / 04 / 07$ & - & -0.17 & -0.05 & -0.12 \\
$2009 / 04 / 08$ & - & -0.20 & -0.05 & -0.12 \\
$2009 / 04 / 09$ & - & -0.21 & -0.06 & -0.14 \\
$2009 / 04 / 14$ & - & -0.19 & -0.06 & -0.12 \\
$2009 / 05 / 05$ & - & -0.14 & -0.04 & -0.10 \\
$2009 / 08 / 05$ & -0.15 & -0.23 & -0.06 & -0.10 \\
$2009 / 08 / 26$ & -0.10 & -0.21 & -0.07 & -0.13 \\
$2009 / 09 / 24$ & - & -0.15 & -0.06 & -0.13 \\
$2010 / 08 / 07$ & - & -0.30 & -0.07 & -0.22 \\
$2010 / 08 / 16$ & -0.18 & -0.36 & -0.08 & -0.27 \\
$2010 / 09 / 14$ & -0.22 & -0.39 & -0.10 & -0.31 \\
\hline
\end{tabular}

includes the Poissonian component and the transformation to the standard Landolt system). They are of a few $\times 0.01-0.001 \mathrm{mag}$.

In addition, classical photoelectric $U B V$ measurements were performed by a single-channel photometer mounted in the Cassegrain focus of 0.6-m reflector at the Skalnate Pleso observatory. Internal uncertainties of these one-day-mean measurements are of a few $\times 0.01 \mathrm{mag}$. These data are presented in Table 3.

Arbitrary flux units of the high-resolution spectra were converted to absolute fluxes with the aid of the simultaneous $U B V R_{\mathrm{C}} I_{\mathrm{C}}$ photometry. To determine flux-points of the true continuum from the measured magnitudes, we calculated corrections for emission lines, $\Delta m_{l}$ (see Skopal 2007), using our lowresolution spectra (Table 4). Then we interpolated these values to dates of the high-resolution spectra. Spectroscopic observations were dereddened with $E_{B-V}=0.27$ (Kenyon \& Webbink 1984) and the resulting parameters were scaled to a distance of $1.73 \mathrm{kpc}$ (Skopal 2000).

\section{Analysis and results}

\subsection{Photometric evolution}

\subsubsection{Indications of a new active phase}

According to the LC evolution from 1988 (Fig. 1), the increase in the star's brightness by $\Delta U \sim 0.5 \mathrm{mag}$ from 2007 July signals that AX Per entered a new active phase. An additional rapid brightening by $\Delta B \sim 0.8 \mathrm{mag}$ and a bluer index $B-V \sim 0.8$, observed during 2009 March (Munari et al. 2009a; and Fig. 2 here), supported the new activity of AX Per. A significant change of the broad minima, observed during quiescent phases at/around the giant inferior conjunction, into a narrower and deeper eclipse, indicates the active phase of a symbiotic binary as well (e.g. Belyakina 1979, 1991; and Figs. 1 and 2 here). During the 2007 conjunction we measured a relatively small, V-type minimum, which position was shifted from the giant conjunction by $\sim-0.025 P_{\text {orb }}$. During the following conjunction the eclipse was deeper and broader, nearly rectangular in profile and placed at the inferior conjunction of the giant. The light observed during the totality became redder with $B-V \sim 1.4$ (see Fig. 2). 

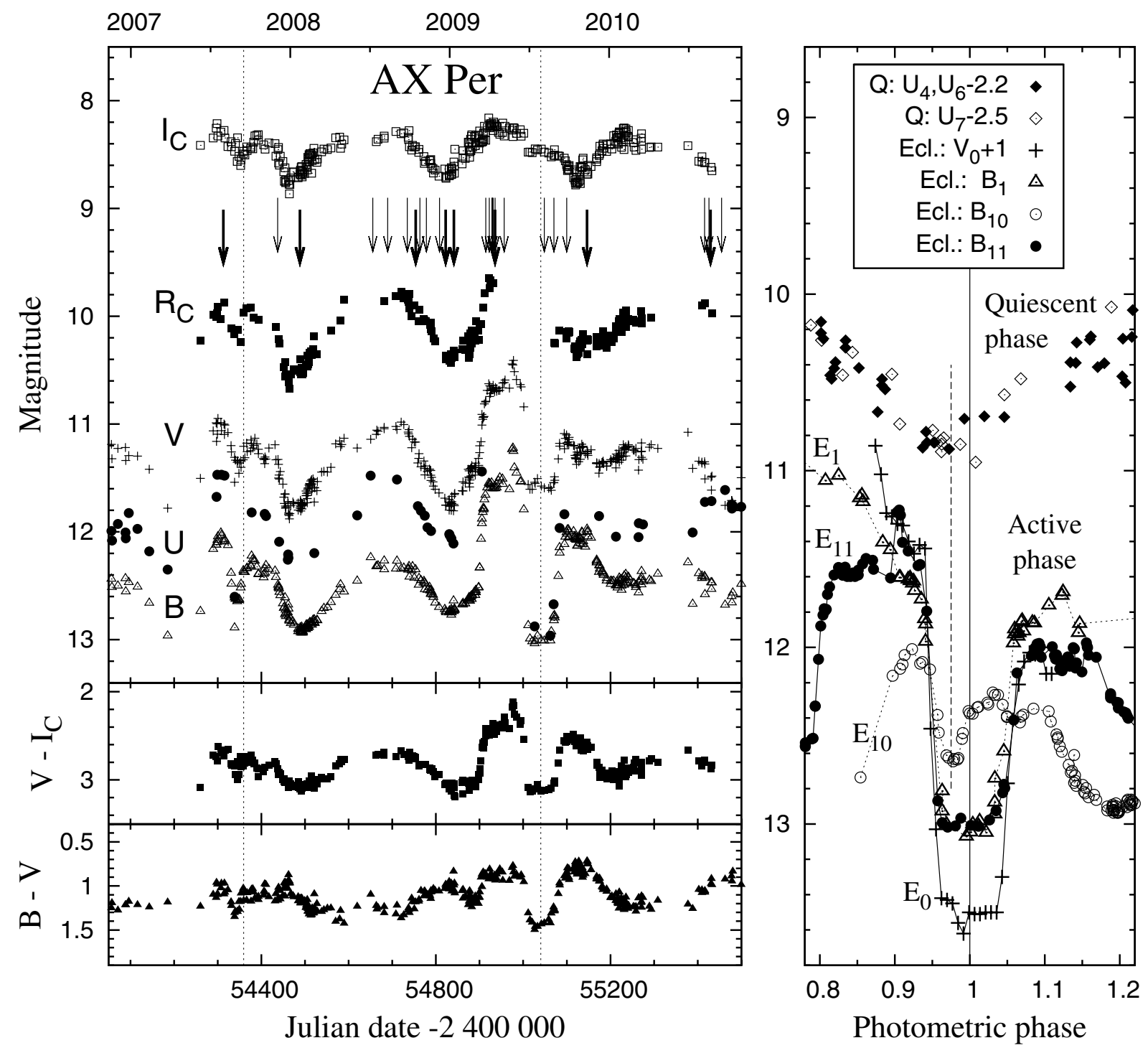

Fig. 2. Left: recent $U B V R_{\mathrm{C}} I_{\mathrm{C}}$ LCs of AX Per covering its 2007-10 active phase. Thin and thick arrows denote the dates of our low- and highresolution spectra, respectively (Table 1). Vertical dotted lines mark the time of eclipses according to Eq. (1). Right: minima profiles selected from LCs in Fig. 1, and folded with the ephemeris (1). Vertical dashed and solid line mark the light minima during quiescent and active phases, respectively. Denotation in keys, for example, "Q: $U_{4}-2.2$ ", means quiescent phase, $U$ filter, epoch 4 and a shift by -2.2 mag.

Finally, we measured a light wave in the LC with a period of $\sim 0.5 P_{\text {orb }}$ and minima located around orbital phases 0.2 and 0.7 (Fig. 2, left). Its presence was transient, being connected only with the 2007-10 higher level of the star's activity (see Fig. 1). It can be seen in all filters, but especially in the $I_{\mathrm{C}}$ band is well pronounced also after the 2009 eclipse (see Fig. 2). This feature was observed in a symbiotic system for the first time. Its understanding requires a detailed analysis, which is, however, out of the scope of this paper. Therefore we postpone it to a future work.

\subsubsection{Eclipses}

The minimum (eclipse), observed during the 2009 brightening, was very similar in profile to those observed during the major 1988-91 active phase. Its well pronounced profile closely covered by measurements, mainly in the $B$ - and $V$-bands, allowed us to determine its contact times as $T_{1}=\mathrm{JD} 2454998.50 \pm 0.79$, $T_{2}=$ JD $2455012.50 \pm 0.53, T_{3}=$ JD $2455065.40 \pm 0.85$ and $T_{4}=$ JD $2455085.33 \pm 0.50$, where uncertainties include only those given by the data coverage, assuming a linear dependence of the star's brightness during the ingress to and ascent from the totality. We neglected other possible sources of errors. These times and their uncertainties indicate an asymmetric profile with $T_{2}-T_{1}=14 \pm 1.3 \mathrm{~d}$ and $T_{4}-T_{3}=$ $20 \pm 1.3 \mathrm{~d}$, which implies an increase of the linear size of the eclipsed object during the totality. Therefore, we determined the middle of the eclipse as the average of all contact times, i.e. $J_{\text {Ecl. }}(2009)=$ JD $2455040.43 \pm 0.67$. Using well-defined timings of other eclipses, JD $\mathrm{Jcl}_{\text {Ecl. }}(1989)=\mathrm{JD} 2447551.7 \pm 1.0$ and $\operatorname{JD}_{\text {Ecl. }}(1990)=$ JD $2448231.6 \pm 0.6($ Skopal 1991), gives their ephemeris as

$\mathrm{JD}_{\text {Ecl. }}=2447551.26( \pm 0.3)+680.83( \pm 0.11) \times E$.

Our photometric ephemeris agrees within uncertainties with that given by the solution of the spectroscopic orbit, $T_{\text {sp.conj. }}=$ $2447553.3( \pm 5.6)+682.1( \pm 1.4) \times E$, obtained from infrared radial velocities by Fekel et al. (2000). Finally, assuming the orbital inclination $i=90^{\circ}$ (see a summary in Skopal et al. 2001) and the separation between the binary components, $a=364 R_{\odot}$ 

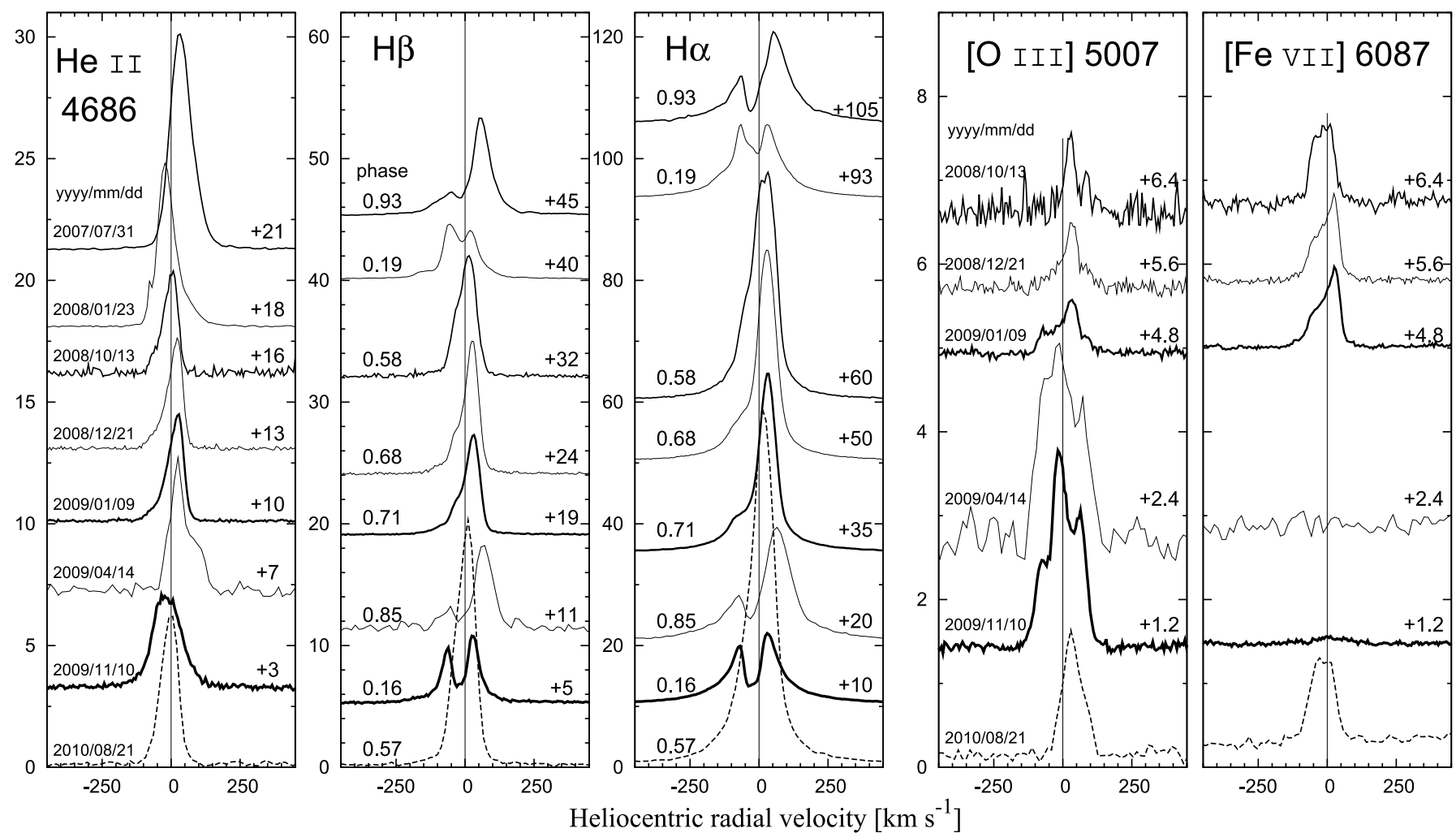

Fig. 3. Evolution in the selected line profiles along the 2007-10 active phase as observed in our high-resolution spectra. Fluxes are in units of $10^{-12} \mathrm{erg} \mathrm{cm}^{-2} \mathrm{~s}^{-1} \AA^{-1}$. Systemic velocity of $-117.44 \mathrm{~km} \mathrm{~s}^{-1}$ was subtracted. Small numbers at the right side of panels represent a shift of profiles with respect to the level of the local continuum. The spectra are dereddened.

(from the mass ratio and the mass function published by Mikolajewska \& Kenyon 1992; Fekel et al. 2000), the radius of the giant, $R_{\mathrm{g}}=115 \pm 2 R_{\odot}$ and that of the eclipsed object, $R_{\mathrm{e}}=27 \pm 2 R_{\odot}$. This result suggests an expansion of the giant radius by $\sim 13 R_{\odot}$, from $102 \pm 3 R_{\odot}$, given by the contact times of the 1990 eclipse (Skopal 1994). The asymmetry of the 2009 eclipse profile and the radius of $R_{\mathrm{e}}=36 \pm 3 R_{\odot}$ derived from the 1990 eclipse profile suggest that the eclipsed object is subject to variation (see also Table 8 below).

\subsection{Spectroscopic evolution}

\subsubsection{The line spectrum}

During the investigated period of the 2007-10 activity, the optical spectrum of AX Per was dominated by $\mathrm{H}_{\mathrm{I}}, \mathrm{He}_{\mathrm{I}}$ and He II emission lines, although some faint emissions, produced by permitted transitions in ionized metals, as e.g. N III 4641 and C III $4647 \AA$, were also present. The spectrum of the forbidden lines was characterized mainly by nebular lines of [O III], [Ne III] and two [Fe vII] lines at 5721 and $6087 \AA$. The Ramanscattered O vi 1032 line at $6825 \AA$ was not present at all. Figure 3 shows evolution in the line profiles of $\mathrm{He}$ II $4686 \AA, \mathrm{H} \beta, \mathrm{H} \alpha$, [O III] $5007 \AA$ and [Fe VII] $6087 \AA$, as observed on our highresolution spectra. Figure 4 demonstrates variations in the line fluxes along the active period, which we observed in all our spectra. Their quantities are introduced in Table 5. We also measured radial velocities (RVs) of main emission peaks in the line profiles of highly ionized elements (Table 6). Figure 5 displays their values compared to the radial velocity curves derived by other authors.
Table 6. Radial velocities in $\mathrm{km} \mathrm{s}^{-1}$ of the main emission peak of highly ionized elements.

\begin{tabular}{lccc}
\hline \hline Date & He II $\lambda 4686$ & {$[\mathrm{Fe}$ VII] $\lambda 6087$} & {$\left[\mathrm{O}_{\text {III }}\right] \lambda 5007$} \\
\hline $2007 / 07 / 31$ & 10.5 & - & - \\
$2008 / 01 / 23$ & 2.0 & - & - \\
$2008 / 10 / 13$ & 6.9 & 10.3 & 27.1 \\
$2008 / 12 / 21$ & 20.3 & 24.8 & 33.4 \\
$2009 / 01 / 09$ & 22.7 & 25.8 & 31.7 \\
$2009 / 04 / 14$ & 23.8 & - & -16.2 \\
$2009 / 11 / 10$ & -18.0 & - & -14.1 \\
$2010 / 08 / 21$ & -0.6 & -13.4 & 29.4 \\
\hline
\end{tabular}

Notes. The systemic velocity was subtracted.

Hydrogen lines: Profiles of hydrogen Balmer lines were significantly affected by a blue-shifted absorption component. Its presence can be recognized around the whole orbital cycle. From the orbital phase $\varphi=0.85$ to $\varphi=1.19$, it created a doublepeaked profile (Fig. 3). In contrast, during the previous quiescent phase, the $\mathrm{H} \alpha$ line observed at similar orbital phases $(\varphi=0.81$ and $\varphi=0.16$, as on 1998 Jan. 9 and Sep. 5, respectively, see Fig. 5 in Skopal et al. 2001), did not display the doublepeaked profile. In addition, both the total flux and the extension of the broad wings of mainly the $\mathrm{H} \alpha$ profile were significantly higher than those measured during quiescent phase (Fig. 6 and Sect. 3.2.2 below). It is important to note that during the major 1988-91 active phase of AX Per a strong absorption cut the broad emission profile even at positions with the hot star in front (see Figs. 5 and 8 of Ivison et al. 1993). This indicates the presence of neutral material along the line of sight around the whole orbit, which attenuates line photons via the $b-b$ transitions. The 


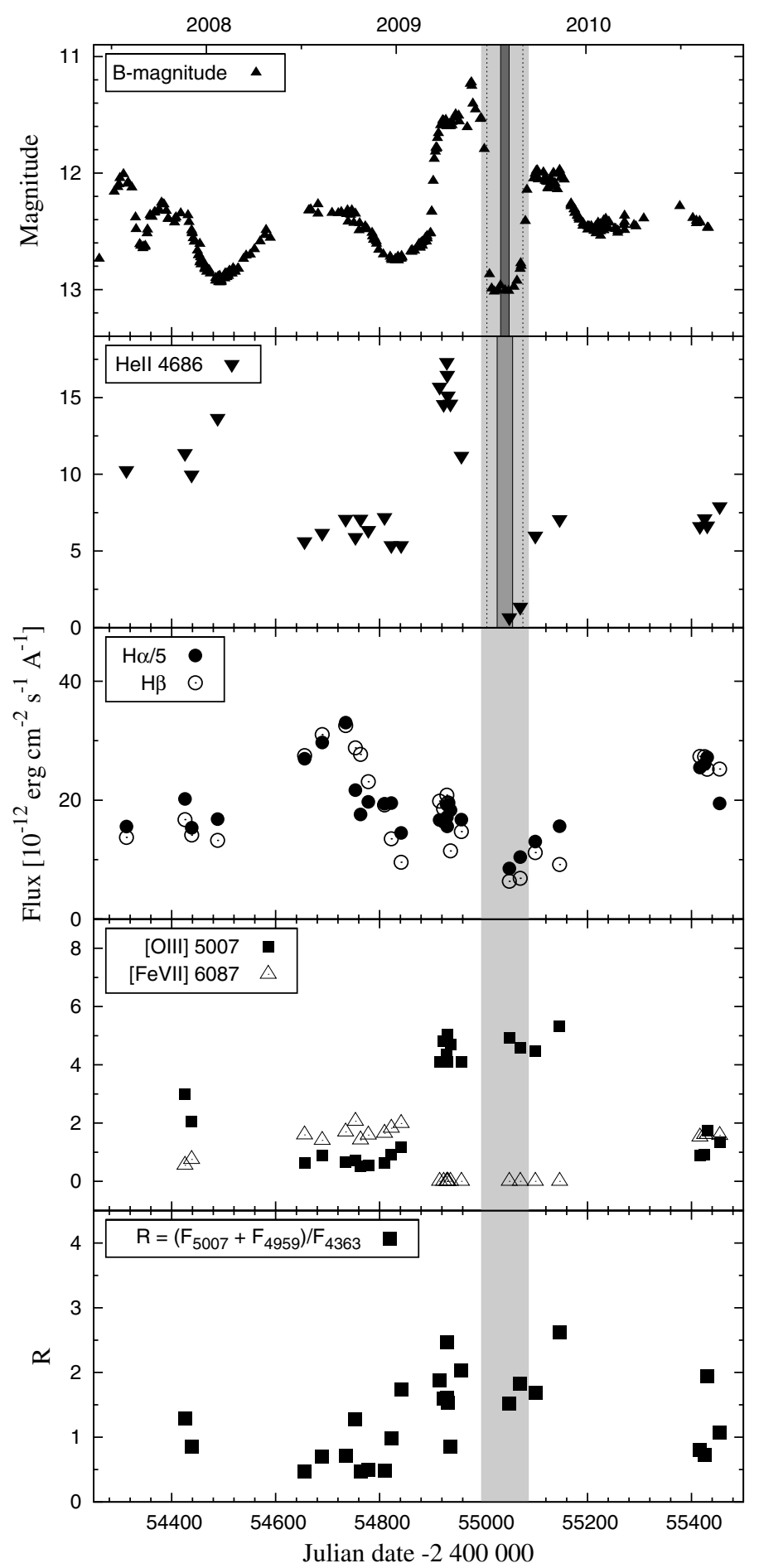

Fig. 4. Variation in the selected line fluxes during the 2007-10 active phase. Top panel shows the $B$-band LC to compare variation in the continuum and to visualize better the time-interval of the eclipse (the light shadow band). The width of the dark and mild shadow band corresponds to the linear size of the eclipsed object and the $\mathrm{He}^{++}$zone, respectively. Dotted lines limit the radius of the cool giant. Fluxes are dereddened and their values are given in Table 5.

strong difference in the line profiles measured during active and quiescent phases suggests that the density of the absorbing material enhances during active phases at the orbital plane. Finally, during the eclipse the total fluxes of $\mathrm{H} \alpha$ and $\mathrm{H} \beta$ lines and those of their wings decreased by a factor of $>3$ with respect to their maximum values (Fig. 4, Table 5). The same behavior was observed

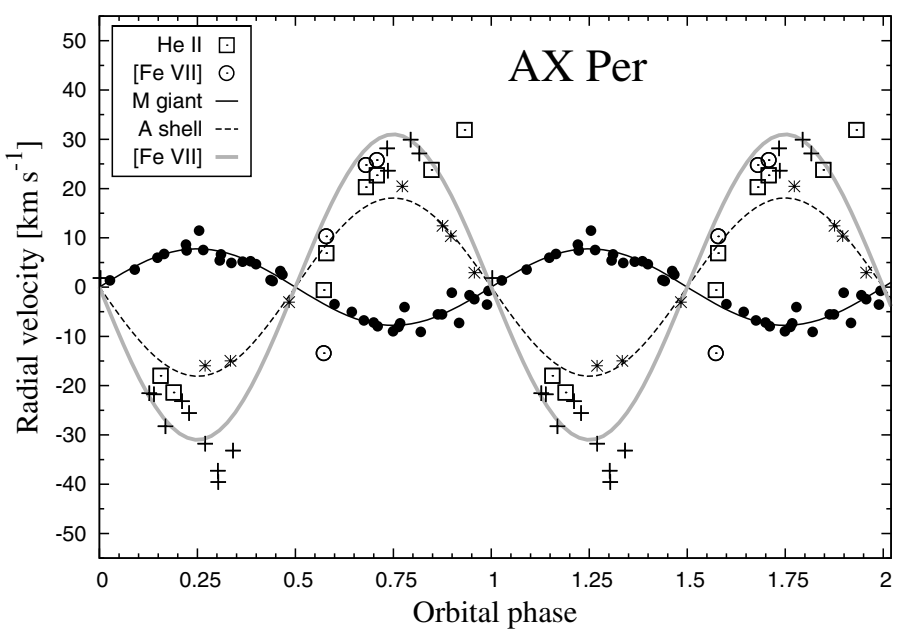

Fig. 5. Radial velocities of main emission peaks measured for highly ionized elements (open symbols, Table 6) as a function of the photometric phase (1). Compared are radial velocity curves for the M-type (Fekel et al. 2000, solid line, filled circles) and A-type absorption lines (Mikolajewska \& Kenyon 1992, dashed line, asterisks), and emission peaks of [Fe VII] $\lambda 6087$ line (Iijima 1988, grey line, crosses). Systemic velocity of $-117.44 \mathrm{~km} \mathrm{~s}^{-1}$ was subtracted.

during the 1994 eclipse (see Fig. 6 of Skopal et al. 2001). Hence, during the active phases, most of the ionized region around the hot star is confined in a region that has about the size of the red giant's radius.

He ॥ $4686 \AA$ line: The He II $4686 \AA$ emission line nearly disappeared during the eclipse. This locates the region of its origin in the hot star vicinity and constrains its maximum linear size to the diameter of the eclipsing giant. The measured flux in the He II 4686 line, $F_{4686}$, allows us to estimate the mean electron concentration in the $\mathrm{He}^{++}$zone. Its luminosity can be expressed as

$4 \pi d^{2} F_{4686}=\alpha_{4686}\left(T_{\mathrm{e}}\right) n_{\mathrm{e}} n\left(\mathrm{He}^{++}\right) h v_{4686} V_{4686}$,

where $\alpha_{4686}\left(T_{\mathrm{e}}\right)$ is the effective recombination coefficient for the given transition, $n_{\mathrm{e}}$ and $n\left(\mathrm{He}^{++}\right)$is the mean concentration of electrons and $\mathrm{He}^{++}$ions, respectively, and $V_{4686}$ is the volume of the $\mathrm{He}^{++}$zone. We also assume that all $\mathrm{He}$ atoms are fully ionized within the zone, i.e. the abundance $A\left(\mathrm{He}^{++}\right)=A(\mathrm{He})$, and thus $n\left(\mathrm{He}^{++}\right)=A(\mathrm{He}) n_{\mathrm{p}}$, because the concentration of hydrogen atoms is equal to that of protons, $n_{\mathrm{p}}$, in the ionized zone. Then the electron concentration

$n_{\mathrm{e}}=\left(1+2 A\left(\mathrm{He}^{++}\right)\right) n_{\mathrm{p}}$,

because each $\mathrm{He}$ atom produces two electrons within the $\mathrm{He}^{++}$ region. For $A\left(\mathrm{He}^{++}\right)=0.1$ the ratio $n_{\mathrm{p}} / n_{\mathrm{e}} \sim 0.83$, and Eq. (2) can be expressed as

$4 \pi d^{2} F_{4686}=\left(\frac{4}{3} \pi R_{\mathrm{g}}^{3} \epsilon\right) \alpha_{4686}\left(T_{\mathrm{e}}\right) A(\mathrm{He}) n_{\mathrm{e}}^{2} 0.83 h v_{4686}$,

where we approximated the $V_{4686}$ volume by a sphere with the radius of the eclipsing giant, $R_{\mathrm{g}}$, and $\epsilon$ is the filling factor. Our measured fluxes, $F_{4686} \sim 1.56 \times 10^{-11}$ and $\sim 7 \times 10^{-12} \mathrm{erg} \mathrm{cm}^{-2} \mathrm{~s}^{-1}$, produced by the $\mathrm{He}^{++}$zone before and after the eclipse, respectively (Fig. 4, Table 5), $\alpha_{4686}(30000 \mathrm{~K}) h v_{4686}=4.2 \times 10^{-25} \mathrm{erg} \mathrm{cm}^{3} \mathrm{~s}^{-1}$ (Hummer \& Storey 1987), $R_{\mathrm{g}}=115 R_{\odot}$ (Sect. 3.1.2.) and $\epsilon=1$, yield a mean $n_{\mathrm{e}}=8.6 \times 10^{9}$ and $5.7 \times 10^{9} \mathrm{~cm}^{-3}$ during the 2009 brightening, before and after the eclipse, respectively. These quantities 
represent a minimum $n_{\mathrm{e}}$, because an optically thin case is assumed. The size of the $\mathrm{He}^{++}$zone can also be estimated from spectroscopic observations made close to the $T_{3}$ time (spectrum 2009/08/26) and just after the $T_{4}$ time (2009/09/24), when the He II 4686 line also aroused from the eclipse (Fig. 4). This time interval ( $\sim 30$ days) corresponds to $R_{4686} \sim 50 R_{\odot}$, which yields $n_{\mathrm{e}} \sim 2.0 \times 10^{10} \mathrm{~cm}^{-3}$ (volume of the eclipsed object was not subtracted).

Profile of the He II $4686 \AA$ line was not symmetrical with respect to the reference wavelength. The RV of its peak was placed mostly at the antiphase to the RV curve of the giant (Fig. 5), which supports the connection of the $\mathrm{He}^{++}$zone to the hot star surroundings. Its larger amplitude indicates an extension of the $\mathrm{He}^{++}$zone to farther distances from the hot star. However, the position of the main core of the profile, measured around the half of its maximum, was shifted blueward relative to the systemic velocity (see also Fig. 5 of Mikolajewska \& Kenyon 1992). The violet shift of the He II $4686 \AA$ emission line was measured also in the spectra of other symbiotics. For example, Tamura et al. (2003) measured it for Z And and Munari et al. (2009b) for AG Dra. Skopal et al. (2006) interpreted this effect by the presence of a disk around the hot star, which blocks a part of the radiation from the wind at its backside to the direction of the observer. Finally, the profile seems to be affected by a variable absorption from its violet side, similarly as for the hydrogen lines (Fig. 3), which makes it difficult to interpret the He II $4686 \AA$ line profile more accurately.

Forbidden lines: In contrast to the $\mathrm{H}$ and $\mathrm{He}$ lines, the nebular $N_{1}$ and $N_{2}$ ([O III] 5007 and $4959 \AA$ ) lines were not subject to the eclipse. Fluxes of the stronger $5007 \AA$ line were as low as $\approx 1 \times 10^{-12} \mathrm{erg} \mathrm{cm}^{-2} \mathrm{~s}^{-1}$ before 2009 March, when they increased to $\sim 4.5 \times 10^{-12} \mathrm{erg} \mathrm{cm}^{-2} \mathrm{~s}^{-1}$, following the increase in the optical continuum. However, during the eclipse the line flux persisted at the same level. In 2010 it returned to the prebrightening values (Fig. 4). The ratio $R=F\left(N_{1}+N_{2}\right) / F_{4363}$, which is a well-known probe of $n_{\mathrm{e}}$ and $T_{\mathrm{e}}$ in planetary nebulae, was extremely low. The observed values, $0.5-2$, imply a high-density $\left[\mathrm{O}_{\mathrm{III}}\right]$ nebula with $n_{\mathrm{e}}([\mathrm{O} \mathrm{III}]) \sim 10^{7}-10^{8} \mathrm{~cm}^{-3}$ and $T_{\mathrm{e}}([\mathrm{O}$ III $])<20000 \mathrm{~K}$ (e.g. Fig. 15.1 in Gurzadyan 1997). A detailed application of the method to AX Per can be found in Skopal et al. (2001), who derived the upper limit of $n_{\mathrm{e}}$ ([O III]) as $7 \times 10^{7} \mathrm{~cm}^{-3}$ for $R=1.7-4.3$. It is interesting that during the 1994 eclipse fluxes in the $N_{1}$ and $N_{2}$ lines faded by a factor of $\leq 2$ with respect to their out-of-eclipse values (see their Fig. 6 and Table 4), in contrast to the evolution during the 2009 eclipse. We discuss this effect in Sect. 4.1.1.

The highly ionized [Fe vII] $6087 \AA$ line was present in the spectrum only during a lower activity level. From the optical brightening in 2009 March to our observation on 2009 November 10th, it entirely disappeared from the spectrum (Table 5). Its profile was asymmetrical with respect to the reference wavelength, being broadened more to the violet side of the line (Fig. 3). As a result, the RV of the intermediate point at half of the line intensity was negative relative to the systemic velocity $\left(-18.1,-9.0,-7.3\right.$ and $-13.4 \mathrm{~km} \mathrm{~s}^{-1}$ as on 2008/10/13, 2008/12/21, 2009/01/09 and 2010/08/21, respectively). However, the peak of the emission line followed the RV curve as derived by Iijima (1988; see Fig. 5 here), which implies that a part of the [Fe vII] zone has to be connected with the hot star, but it is located farther from it, behind the binary. The [Fe VII $]$ transitions are excited by collisions with thermal electrons (Nussbaumer \& Osterbrock 1970). The enhanced ionized wind during the brightening produces a larger amount of free

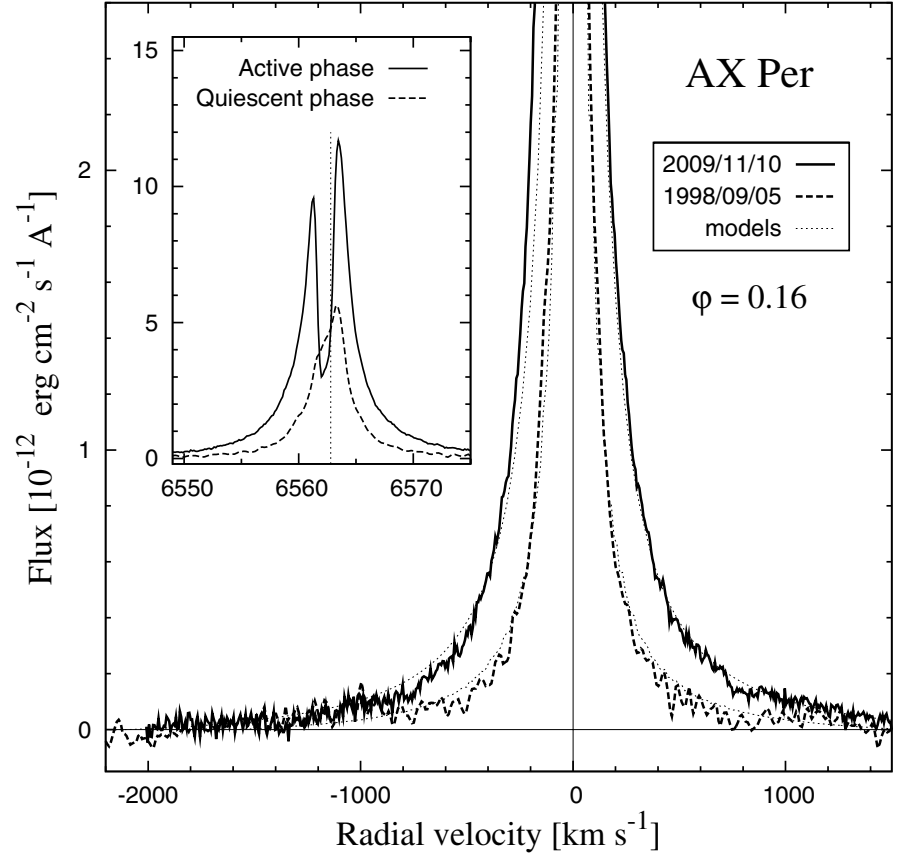

Fig. 6. Example of the broad $\mathrm{H} \alpha$ wings from the 2007-10 active phase (solid line), compared with those observed during quiescent phase (dashed line). Both spectra were taken at the same orbital phase, $\varphi=$ 0.16 . Local continua were subtracted from the spectra. The spectra were dereddened. The models (dotted lines) indicate a significant enhancement of the mass-loss rate during activity with respect to quiescence (Table 7, Sect. 3.2.2).

electrons, while the [Fe vII] $6087 \AA$ emission line disappears. This anti-correlation can be understood if the [Fe VII] region is placed instead at the orbital plane, where the material is in the ionization shadow during the phase of a warm disk creation (see Sect. 4). During quiescence, when the disk is not present, the [Fe VII] line can be created within the collisional zone of winds from the binary components (Wallerstein et al. 1984). According to its shaping in the binary (see Fig. 5 of Bisikalo et al. 2006), a fraction of the [Fe VII] emission could arise behind the binary from the hot star side, and thus satisfy the RV of its orbital motion with a larger amplitude (see Fig. 5). However, more highresolution observations of the [Fe vII] $6087 \AA$ line for eclipsing symbiotic binaries are needed to understand its origin in symbiotic systems better.

\subsubsection{Mass loss through the hot star wind}

The hot components in symbiotic binaries can lose their mass in the form of wind (e.g. Vogel 1993; Nussbaumer et al. 1995; Eriksson et al. 2004; Kenny \& Taylor 2005), which enhances considerably during active phases (Skopal 2006). To estimate its rate, we used the $\mathrm{H} \alpha$ method as proposed by Skopal (2006), which assumes that the broad $\mathrm{H} \alpha$ wings originate in the ionized wind from the hot star. The principle of the method is a comparison of the observed and synthetic profile of the broad wings.

The model assumes a spherically symmetric wind originating in the central star, which is covered by an optically thick disk in the direction of the observer. The disk is characterized with the radius $R_{\mathrm{D}}$ and height $H_{\mathrm{D}}$, and it is seen edge-on in the model (see Fig. 9). Density, and thus the emissivity of the wind at a given distance $r$ from the hot star centre, is determined by the massloss rate and the velocity of the wind. The velocity distribution 
Table 7. Parameters of synthetic models of the broad $\mathrm{H} \alpha$ wings $\left(R_{\mathrm{w}}\right.$, $\left.\beta, v_{\infty}\right)$, their luminosities observed for $|R V| \geq 200 \mathrm{~km} \mathrm{~s}^{-1}, L_{\alpha}(200)$, and corresponding mass-loss rates, $\dot{M}_{\mathrm{w}}$.

\begin{tabular}{lccccc}
\hline \hline $\begin{array}{l}\text { Date } \\
\text { dd/mm/yyyy }\end{array}$ & $\begin{array}{c}R_{\mathrm{w}} \\
{\left[R_{\odot}\right]}\end{array}$ & $\beta$ & $\begin{array}{c}v_{\infty} \\
{\left[\mathrm{km} \mathrm{s}^{-1}\right]}\end{array}$ & $\begin{array}{c}L_{\alpha}(200) \\
{\left[L_{\odot}\right]}\end{array}$ & $\begin{array}{c}\log \left(\dot{M}_{\mathrm{w}}\right) \\
{\left[M_{\odot} \mathrm{yr}^{-1}\right]}\end{array}$ \\
\hline \multicolumn{6}{c}{ Quiescent phase: $R_{\mathrm{D}}=0.32 R_{\odot}, H_{\mathrm{D}}=0.032 R_{\odot}$} \\
$1998 / 01 / 09$ & 0.027 & 1.75 & 1500 & 0.90 & -7.04 \\
$1998 / 09 / 05$ & 0.030 & 1.75 & 1600 & 0.88 & -7.02 \\
Active phase: $R_{\mathrm{D}}=20 R_{\odot}, H_{\mathrm{D}}=6 R_{\odot}$ & \\
$2007 / 07 / 31$ & 5.20 & 1.75 & 2500 & 2.97 & -5.52 \\
$2008 / 01 / 23$ & 5.27 & 1.74 & 2500 & 2.13 & -5.59 \\
$2008 / 10 / 13$ & 5.40 & 1.75 & 1500 & $0.83^{\dagger}$ & -5.84 \\
$2008 / 12 / 21$ & 5.45 & 1.72 & 2000 & 1.60 & -5.70 \\
$2009 / 01 / 09$ & 5.40 & 1.75 & 2000 & 1.40 & -5.72 \\
$2009 / 04 / 14$ & 5.25 & 1.73 & 2500 & 3.17 & -5.49 \\
$2009 / 11 / 10$ & 5.20 & 1.75 & 2100 & $1.42^{\dagger}$ & -5.67 \\
$2010 / 08 / 21$ & $0.94^{\dagger}$ & 1.80 & 2000 & $2.25^{\dagger}$ & -5.96 \\
\hline
\end{tabular}

Notes. ${ }^{\dagger}|R V|=250 \mathrm{~km} \mathrm{~s}^{-1},{ }^{\ddagger} R_{\mathrm{D}}=3.5 R_{\odot}, H_{\mathrm{D}}=1.05 R_{\odot}$.

is approximated with (Castor et al. 1975)

$v(r)=v_{\infty}\left(1-R_{\mathrm{w}} / r\right)^{\beta}$,

where the origin of the wind, $R_{\mathrm{w}}$, and $\beta$ are model parameters, while the terminal velocity, $v_{\infty}$, is given by the extension of the wings. During the quiescent phase, parameters $R_{\mathrm{D}}$ and $H_{\mathrm{D}}$ can be estimated from the effective radius of the hot star, $R_{\mathrm{h}}^{\mathrm{eff}} \sim 0.1 R_{\odot}$ (Table 3 of Skopal 2005). Assuming the ratio $H_{\mathrm{D}} / R_{\mathrm{D}}=0.1$ yields $R_{\mathrm{D}}=0.32 R_{\odot}$ and $H_{\mathrm{D}}=0.032 R_{\odot}$. During the investigated active phase, we estimated the disk radius from the first two contact times of the 2009 eclipse (Sect. 3.1.2) as $R_{\mathrm{D}} \sim 20 R_{\odot}$. Assuming a disk with $H_{\mathrm{D}} / R_{\mathrm{D}}=0.3$, yields $H_{\mathrm{D}}=$ $6 R_{\odot}$. We recall here that the results do not critically depend on these parameters. The mass-loss rate is mainly determined by the luminosity of the broad wings and their terminal velocity (see Eq. (14) of Skopal 2006). A comparison between the modeled and observed profiles is shown in Fig. 6. The synthetic profiles match the observed wings well for $|R V| \gtrsim 200-250 \mathrm{~km} \mathrm{~s}^{-1}$. There is a significant difference between the extension and luminosity of the $\mathrm{H} \alpha$ wings observed during the quiescent and active phase (Table 7, Fig. 6). Broad wings from the 1998 quiescence corresponded to the mass-loss rate of $\lesssim 1 \times 10^{-7} M_{\odot} \mathrm{yr}^{-1}$, while during the activity it increased to $\approx 2-3 \times 10^{-6} M_{\odot} \mathrm{yr}^{-1}$.

In our model the mass-loss rate determines the particle density at a given distance $r$ from the origin of the wind according to the continuity equation as

$n_{\mathrm{w}}(r)=\dot{M}_{\mathrm{w}} / 4 \pi r^{2} \mu m_{\mathrm{H}} v(r)$,

where the wind velocity, $v(r)$, is given by Eq. (5), $\mu$ is the mean molecular weight and $m_{\mathrm{H}}$ is the mass of the hydrogen atom. Within the $\mathrm{He}^{++}$zone, i.e. from the hot star to $r_{\mathrm{HeII}} \approx 50 R_{\odot}$ (Sect. 3.2.1), the particle densities $n_{\mathrm{w}}(r)$ excellently agree with those derived independently of the measured $F_{4686}$ fluxes. For example, $\dot{M}_{\mathrm{w}}=3 \times 10^{-6} M_{\odot} \mathrm{yr}^{-1}$ and $v_{\infty}=2000 \mathrm{~km} \mathrm{~s}^{-1}$, yield $n_{\mathrm{W}}\left(20 R_{\odot}\right) \sim 2.8 \times 10^{10}$ (i.e. $n_{\mathrm{e}}=n_{\mathrm{w}} / 0.83=3.3 \times 10^{10} \mathrm{~cm}^{-3}$ ), and $n_{\mathrm{w}}\left(50 R_{\odot}\right) \sim 3.2 \times 10^{9} \mathrm{~cm}^{-3}\left(n_{\mathrm{e}}=3.9 \times 10^{9} \mathrm{~cm}^{-3}\right)$. This suggests that particles of the hot star wind give rise to the observed emission from the $\mathrm{He}^{++}$zone.

\subsubsection{Modeling the continuum spectrum}

The observed continuum spectrum of symbiotic stars, $F(\lambda)$, can formally be expressed as a superposition of its three basic components (see Sect. 1), i.e.

$F(\lambda)=F_{\mathrm{g}}(\lambda)+F_{\mathrm{n}}(\lambda)+F_{\mathrm{h}}(\lambda)$,

where $F_{\mathrm{g}}(\lambda), F_{\mathrm{n}}(\lambda)$ and $F_{\mathrm{h}}(\lambda)$ represent radiative contributions from the cool giant, nebula and a hot stellar source, respectively. Basic features of these components of radiation are well recognizable in our low-resolution spectra exposed from short wavelengths, $\lambda \gtrsim 3300 \AA$. Most distinctive are molecular passbands arising in the red giant atmosphere, while a pronounced Balmer jump in emission directly indicates a strong nebular continuum. The source of ionizing photons (i.e. the hot star) is indicated indirectly by the presence of strong recombination lines superposed on the continuum. Its direct indication is possible only within the super-soft X-ray and far-UV; in the optical its contribution is negligible (e.g. Fig. 2 of Skopal et al. 2009). Another significantly cooler stellar source of radiation develops during active phases. It is connected with the hot star, and its evidence is given directly by eclipses in the optical LCs (e.g. Belyakina 1979; Skopal 1991) and/or by model SED at any other orbital phase (Skopal 2005). Hereafter we call it a warm stellar component (WSC).

The aim of this section is to disentangle the composite spectrum, observed at different stages of AX Per activity, into its individual components of radiation, i.e. to determine their physical parameters. For the $F_{\mathrm{g}}(\lambda)$ component we used photospheric synthetic spectra of M-giant stars published by Fluks et al. (1994). These models were calculated in the spectral range $350-900 \mathrm{~nm}$ for 11 spectral types (ST), from M0 to M10. In our modeling we determine the ST and its subclass by a linear interpolation between the neighboring best-fitting STs. Because the radiation from the giant can vary, the scaling factor of the synthetic spectra represents another parameter in the model SED.

We approximated the spectrum produced by the nebula by

$F_{\mathrm{n}}(\lambda)=k_{\mathrm{n}} \times \varepsilon_{\lambda}\left(T_{\mathrm{e}}\right)$,

where the scaling factor $k_{\mathrm{n}}\left[\mathrm{cm}^{-5}\right]$ represents the emission measure $(E M)$ of the nebula scaled with the distance $d$, i.e., $E M=4 \pi d^{2} \times k_{\mathrm{n}} . \varepsilon_{\lambda}\left(T_{\mathrm{e}}\right)$ is the volume emission coefficient [erg cm $\mathrm{cm}^{3} \mathrm{~s}^{-1} \AA^{-1}$, which depends on the electron temperature, $T_{\mathrm{e}}$, and is a function of the wavelength (e.g. Brown \& Mathews 1970). By this approach we can register only those photons produced by the optically thin part of the nebular medium, and thus estimate the lower limit of EM. For the sake of simplicity, we calculated the $\varepsilon_{\lambda}\left(T_{\mathrm{e}}\right)$ coefficient for the hydrogen plasma only, including contributions from recombinations and thermal bremsstrahlung. Some additional arguments for this approximation are given in Skopal et al. (2009). In addition, we assumed that $T_{\mathrm{e}}$ and thus $\varepsilon_{\lambda}\left(T_{\mathrm{e}}\right)$ are constant throughout the nebula.

The presence of F-G type features in the spectra before and after the 2009 eclipse suggests that the temperature of the stellar source is as low as $\approx 5000-7000 \mathrm{~K}$. As the most obvious F$\mathrm{G}$ features we identified the broad Ca I $\lambda 4227$ and $\mathrm{Ca}$ II K $\lambda 3934$ line (Fig. 7). Also the absorption at $\lambda 3810$, pronounced mainly in the 2009/04/09 spectrum, is clearly evident in the F and G spectral templates showed in Figs. 218 to 222 of Munari \& Zwitter (2002). Therefore, we compared the continuum of the hot stellar source in Eq. (7) to synthetic spectra, calculated for 5000$10000 \mathrm{~K}$ using the Kurucz's codes (Munari et al. 2005). So, the third term of Eq. (7) can be written as

$F_{\mathrm{h}}(\lambda)=\left(\theta_{\text {warm }}^{\text {eff }}\right)^{2} \mathcal{F}_{\lambda}\left(T_{\text {eff }}\right)$,

where $\mathcal{F}_{\lambda}\left(T_{\text {eff }}\right)$ is the WSC of radiation. Its corresponding effective temperature, $T_{\mathrm{eff}}$, and the scaling factor, $\theta_{\mathrm{warm}}^{\mathrm{eff}}=R_{\mathrm{warm}}^{\mathrm{eff}} / d$, 
Table 8. Parameters of the SED-fitting analysis (see Sect. 3.2.3, Fig. 8).

\begin{tabular}{|c|c|c|c|c|c|c|c|c|c|c|}
\hline \multirow{2}{*}{$\begin{array}{l}\text { Date } \\
\text { yyyy/mm/dd }\end{array}$} & \multirow[t]{2}{*}{ Stage/B-mag } & \multicolumn{3}{|c|}{ Giant } & \multicolumn{3}{|c|}{ Warm stellar object } & \multicolumn{2}{|r|}{ Nebula } & \multirow[t]{2}{*}{$\chi_{\text {red }}^{2}$} \\
\hline & & ST & $T_{\text {eff }} / \mathrm{K}^{\dagger}$ & $L_{\mathrm{g}} / L_{\odot}^{\star}$ & $T_{\text {eff }} / \mathrm{K}$ & $R_{\mathrm{warm}}^{\mathrm{eff}} / R_{\odot}$ & $L_{\mathrm{warm}} / L_{\odot}$ & $T_{\mathrm{e}} / \mathrm{K}$ & $E M / 10^{59} \mathrm{~cm}^{-3}$ & \\
\hline $2007 / 12 / 03$ & $\mathrm{~A} / 12.5$ & M5.8 & 3316 & 1430 & 5250 & 6.2 & 26 & 34000 & 3.5 & 0.75 \\
\hline $2008 / 10 / 23$ & A / 12.4 & M5.8 & 3316 & 1430 & 5250 & 8.2 & 45 & 23000 & 3.8 & 1.17 \\
\hline 2008/12/08 & A / 12.7 & M5.8 & 3316 & 1430 & - & - & - & 32000 & 4.8 & 0.87 \\
\hline $2009 / 04 / 09$ & A / 11.6 & M5.7 & 3326 & 1450 & 5500 & 11 & 98 & 25000 & 8.2 & 0.80 \\
\hline $2009 / 08 / 05$ & $\mathrm{~A} / 13.0^{\ddagger}$ & M5.8 & 3316 & 1430 & - & - & - & 33000 & 2.6 & 0.78 \\
\hline $2009 / 08 / 26$ & $\mathrm{~A} / 12.8^{\ddagger}$ & M5.6 & 3336 & 1470 & - & - & - & 28000 & 1.7 & 1.06 \\
\hline $2009 / 09 / 24$ & $\mathrm{~A} / 12.0$ & M5.6 & 3336 & 1470 & 6250 & 7.0 & 68 & 32000 & 4.0 & 0.55 \\
\hline $2010 / 08 / 16$ & $\mathrm{Q} / 12.4$ & M5.3 & 3366 & 1520 & - & - & - & 27000 & 4.0 & 1.30 \\
\hline 2010/09/14 & $\mathrm{Q} / 12.5$ & M5.3 & 3366 & 1520 & - & - & - & 27000 & 3.5 & 1.67 \\
\hline
\end{tabular}

Notes. ${ }^{\dagger}$ According to the Fluks et al. (1994) calibration, ${ }^{\star}$ for $R_{\mathrm{g}}=115 R_{\odot},{ }^{\star}$ eclipse.

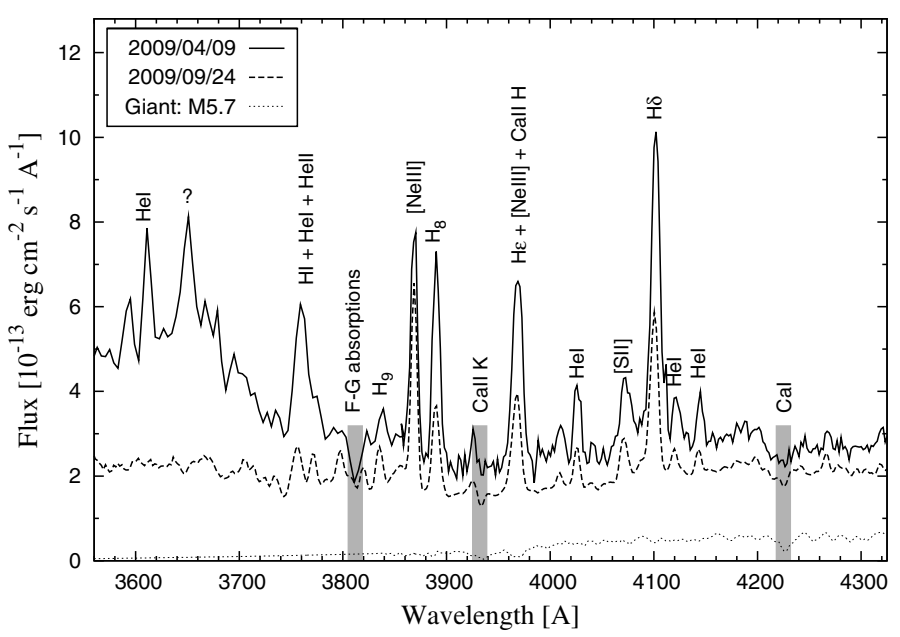

Fig. 7. Blue part of the dereddened spectra with well-developed warm pseudophotosfere as suggested by the model SED (Fig. 8). Features typical for the spectrum of F-G giants are marked by gray bands.

represent free parameters in modeling the SED. Other atmospheric parameters of synthetic spectra were fixed $(\log (g)=$ $2.5,[M / H]=0,[\alpha / \mathrm{Fe}]=0, v_{\text {rot }}=20 \mathrm{~km} \mathrm{~s}^{-1}$ ), and their resolution was accommodated to that of our low-resolution spectra.

After defining the individual components of radiation in Eq. (7), we prepared a grid of models for reasonable ranges of the fitting parameters (ST, its subclass and scaling for the giant; $T_{\mathrm{e}}$ and $k_{\mathrm{n}}$ for the nebular continuum; $T_{\text {eff }}$ and $\theta_{\text {warm }}^{\text {eff }}$ for the WSC), and selected that which corresponded to a minimum of the function

$\chi^{2}=\sum_{\mathrm{i}=1}^{\mathrm{N}}\left[\frac{\left(F^{\mathrm{obs}}\left(\lambda_{\mathrm{i}}\right)-F\left(\lambda_{\mathrm{i}}\right)\right.}{\Delta F^{\mathrm{obs}}\left(\lambda_{\mathrm{i}}\right)}\right]^{2}$,

where $F^{\mathrm{obs}}\left(\lambda_{\mathrm{i}}\right)$ are fluxes of the observed continuum, $N$ is their number (1500-1800), $\Delta F^{\mathrm{obs}}\left(\lambda_{\mathrm{i}}\right)$ are their errors, and $F\left(\lambda_{\mathrm{i}}\right)$ are theoretical fluxes given by Eq. (7). Input flux-points were selected from the observed spectra by omitting emission lines and a spectral region from 3645 to $\sim 3740 \AA$, where hydrogen lines of the high members of Balmer series were blended, which did not allow us to identify the true continuum. For the flux uncertainties we adopted typical values of $10 \%$.

Our resulting models of the AX Per composite continuum along its 2007-10 active phase are shown in Fig. 8 and the corresponding parameters are given in Table 8 . The presence of the WSC in the $3200-7500 \AA$ spectrum is constrained by a low Balmer jump and high values of the observed fluxes in the blue part of the spectrum. To fit the small Balmer discontinuity and to fill in the large difference between the contribution from the giant and the observed spectrum for $\lambda \lesssim 5000 \AA$, an additional radiative component with a fairly flat energy distribution throughout the optical was required. This component satisfies the radiation from a stellar source with temperature of 5000 $6000 \mathrm{~K}$ well, and can accordingly be associated with the WSC. The WSC and the nebular emission were strongest during the 2009 brightening (Table 8). The following eclipse interrupted the brightening by removing the WSC entirely and the nebular component partially from the spectrum (EM decreased by a factor of $\gtrsim 3$ ). Immediately after the eclipse, the WSC arose again in the spectrum together with a stronger nebular emission (Fig. 8 and Table 8). We were able to fit our last spectra from 2010 August and September without the WSC, which signaled the return of AX Per to quiescent phase. For comparison, we inserted the last spectrum into the UV-IR SED from quiescence (adapted according to Fig. 21 of Skopal 2005) to demonstrate the negligible contribution of the hot star in the optical (Fig. 8, bottom).

For comparison, we also derived $T_{\mathrm{e}}, E M$ and the intrinsic $V$-magnitude of the giant, $V_{\mathrm{g}}$, from the observed $U B V$ magnitudes by using the method of Cariková \& Skopal (2010). For the 2009 eclipse we used $B=13.01$ and $V=11.59$, taken simultaneously with the low-resolution spectrum from $2009 / 08 / 05$. The $U$ magnitude was interpolated to 12.93 at this date. After simultaneous corrections for emission lines (Table 4), we determined $T_{\mathrm{e}} \sim 37000 \mathrm{~K}, E M=2.7 \pm 0.2 \times 10^{59}(d / 1.73 \mathrm{kpc})^{2} \mathrm{~cm}^{-3}$ and $V_{\mathrm{g}}=10.98 \pm 0.02$. Uncertainties of the resulting parameters reflect errors of photometric measurements. The $V_{\mathrm{g}}$ flux-point matches the contribution from the giant well (Fig. 8), the EM is also well comparable with that derived from the model SED (Table 8 ), but $T_{\mathrm{e}}$ suffers from a large uncertainty caused by a weak dependence of the nebular continuum profile on $T_{\mathrm{e}}$ within the $U B V$ region only.

\section{Discussion}

From 2007 on, AX Per entered a new active phase (Sect. 3.1.1). This was also supported by a correlation between the visual brightness, EM and the WSC luminosity (see Table 8), as observed for the hot components during active phases in other symbiotic stars (see Table 4 of Skopal 2005). The 2007-10 active phase was connected with a significant change in the ionization structure in the binary during the transition from the preceding quiescent phase. 

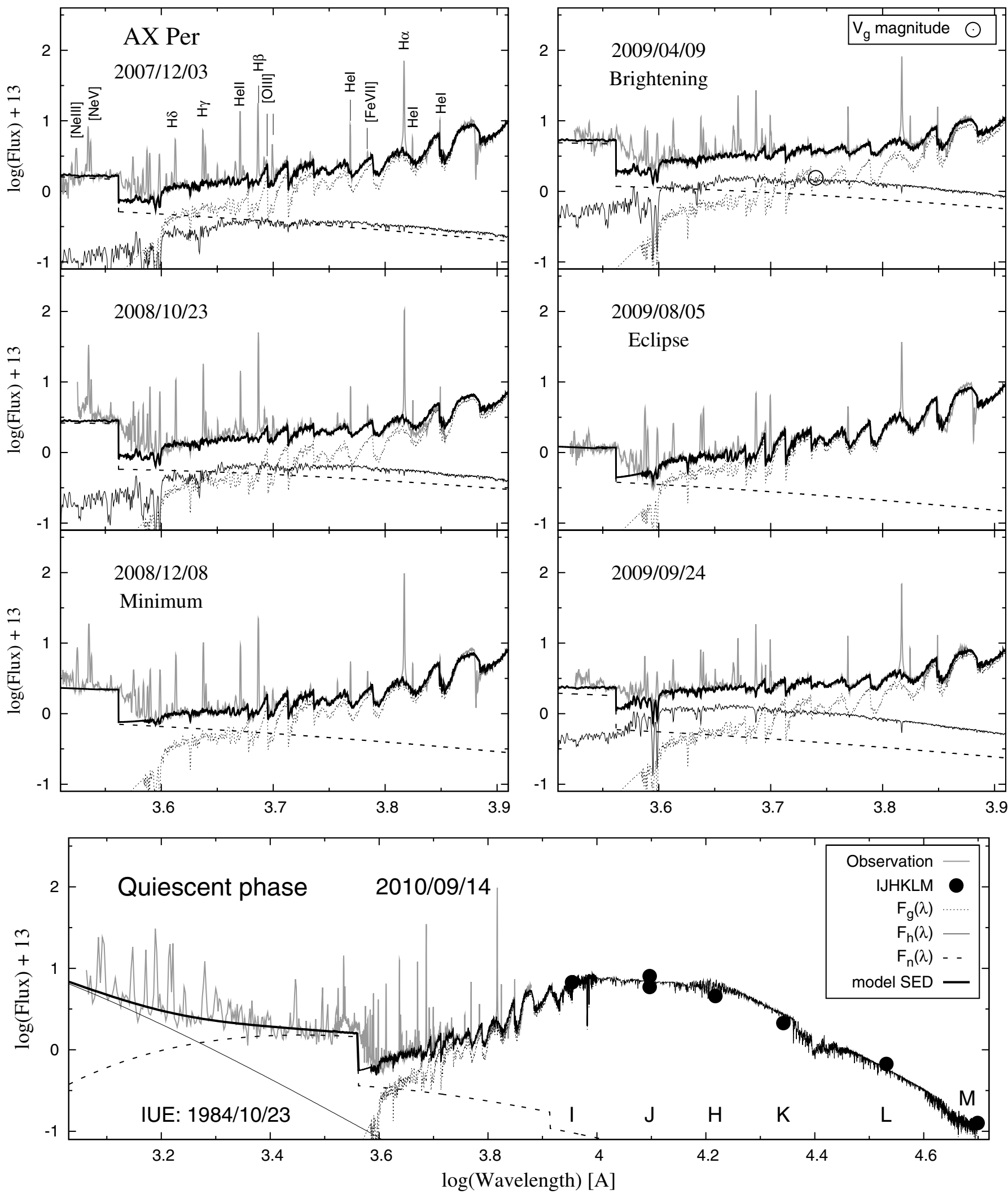

Fig. 8. Our low-resolution dereddened spectra (gray lines) and their models (heavy solid lines) at selected dates during the 2007-10 active phase of AX Per. The model SEDs and their components of radiation here represent a graphic form of Eq. (7) with the same denotation in keys (in the bottom panel). Fluxes are in units $\mathrm{erg} \mathrm{cm}^{-2} \mathrm{~s}^{-1} \AA^{-1}$. Corresponding parameters are collected in Table 8. Modeling is described in Sect. 3.2.3.

(i) In the continuum, this was indicated by a distinctive change in the shape of the minimum observed at/around the inferior conjunction of the giant. During quiescence, the broad minima in the LC (e.g. $E=4$ to 9 in Fig. 1) are caused by the orbital motion of extended ionized wind from the giant, which is partially optically thick (Skopal 2001). During the 
active phase, the broad minima were replaced by narrow ones (eclipses), which suggests that a large part of the optical light produced by the hot component was concentrated at/around the hot star.

(ii) In the line spectrum, we indicated a significant increase in the mass-loss rate via the hot star wind with respect to the quiescent phase (Sect. 3.2.2, Fig. 6, Table 7). For example, during the 2009 brightening, we measured the highest luminosity and extension of the $\mathrm{H} \alpha$ wings, which corresponded to the mass-loss rate of $\sim 3 \times 10^{-6} M_{\odot} \mathrm{yr}^{-1}$.

\subsection{Evolution of the ionized zone}

Throughout the whole $2007-10$ period we observed a strong nebular component of radiation in the spectrum (Fig. 8, Table 8). A geometrical change of the main source of the nebular radiation was indicated by the change in the position and profile of eclipses during the 2007 and 2009 inferior conjunction of the giant. The 2007 minimum was shifted from the conjunction by $\sim-0.025 P_{\text {orb }}$ (Fig. 2, right). This implies that an enhanced emitting region had to be located in front of the hot star, preceding its orbital motion. As discussed by Bisikalo et al. (2006), this higher density region can develop about 100-150 days after an increase of the hot star wind as a result of its collision with the giant's wind and the orbital motion (see their Figs. 4 and 5). This region will be ionized by the neighboring hot star, and thus produce a surplus of nebular emission. As a result, the light minimum will precede the inferior conjunction of the giant. However, after the following orbital cycle, the minimum was located exactly at the giant's conjunction (Fig. 2). Changes in the position of the light minima during different stages of activity were indicated also for other eclipsing symbiotic binaries (see Skopal 1998).

During the 2009.6 eclipse the WSC of radiation disappeared entirely, and the nebular component faded by a factor of $\sim 3$ with respect to the out-of-eclipse values (Table 8, Fig. 8). This implies that a strong source of the nebular continuum had to be located in the vicinity of the hot star, within the region eclipsed by the giant. According to the modeling of the broad $\mathrm{H} \alpha$ wings (Sect. 3.2.2), the eclipsed emission region can be ascribed to the ionized wind from the hot star.

\subsubsection{Nebular lines}

Spectroscopic observations made after the major 1989-91 active phase at/around the 1994 eclipse showed that the nebular [O III] lines were subject to eclipse. Their fluxes faded by a factor of $\sim 2$ with respect to values measured before and after the eclipse (see Table 4 of Skopal et al. 2001). In contrast, from 2009 March, nebular [O III] line fluxes increased by a factor of $\sim 4.5$ and kept their values at a constant level to our last 2009 observation (2009/11/10), i.e. also throughout the eclipse (Fig. 4). This behavior implies that the hot star wind causes a fraction of the $\left[\mathrm{O}_{\mathrm{III}}\right]$ region. Its location is therefore a function of the mass-loss rate, which determines particle densities at a given distance from the wind source. Higher $\dot{M}_{\mathrm{w}}$ places the [O III] zone to longer distances from the hot star and vice versa. During the active phase, $\dot{M}_{\mathrm{W}} \sim 2 \times 10^{-6} M_{\odot} \mathrm{yr}^{-1}, v_{\infty}=2000 \mathrm{~km} \mathrm{~s}^{-1}$ and critical densities for creation of nebular lines, $n_{\mathrm{e}} \gtrsim 10^{7} \mathrm{~cm}^{-3}$, place the [O III] zone at distances $r>210 R_{\odot}$ from the hot star, i.e. far beyond the eclipsed region. However, during quiescence, $\dot{M}_{\mathrm{w}} \sim 1 \times 10^{-7} M_{\odot} \mathrm{yr}^{-1}$ and $v_{\infty}=1600 \mathrm{~km} \mathrm{~s}^{-1}$ (Table 7) correspond to the critical radius of $\sim 50 R_{\odot}$, which thus can be partially eclipsed by the giant with $R_{\mathrm{g}} \sim 115 R_{\odot}$. Finally, the
[O III] line profiles consist of more components (Fig. 3), which suggests that the hot star wind at farther distances from its origin was structured and not simply uniform in direction and density.

\subsection{The neutral zone}

We indicated the presence of a WSC of radiation in the composite spectrum photometrically by the 2009 eclipse profile, and spectroscopically by separating the observed spectrum into its individual components. Its source is characterized with effective temperatures of $\sim 5500-6500 \mathrm{~K}$ and effective radii of $\sim 6-11 R_{\odot}$, which correspond to luminosities between $\sim 30$ and $100 L_{\odot}$ (Table 8). However, its shape cannot be spherical: (i) if this were a sphere, its radiation would not be capable of causing the observed nebular emission. On the other hand, the presence of the strong nebular component in the spectrum constrains the presence of a hot ionizing source in the system; (ii) if the radius of the eclipsed object, $R_{\mathrm{e}}=27 R_{\odot}$ (Sect. 3.1.2), were that of a sphere, its luminosity would be a factor of $\sim 15$ higher than that we observed at the 2009 bright stage. These arguments suggest that the source of the WSC, which is subject to the eclipse, has a form of a disk with the radius $R_{\mathrm{D}}=R_{\mathrm{e}}$. Assuming that its outer rim represents the warm pseudophotosphere (Sect. 5.3.5 of Skopal 2005), then its luminosity

$2 \pi R_{\mathrm{D}} 2 H_{\mathrm{D}} \sigma T_{\mathrm{eff}}^{4}=4 \pi\left(R_{\mathrm{warm}}^{\mathrm{eff}}\right)^{2} \sigma T_{\mathrm{eff}}^{4}$.

For $H_{\mathrm{D}} / R_{\mathrm{D}} \equiv 0.3$ (Sect. 3.2.2) and $R_{\text {warm }}^{\text {eff }}=11 R_{\odot}$ (Table 8) we get $R_{\mathrm{D}}=20 R_{\odot}$, which agrees well with $R_{\mathrm{e}}=22.3 \pm 2 R_{\odot}$, obtained from the first two contact times of the 2009 eclipse. Therefore we conclude that the warm stellar object, which developed during the active phase, has the form of a flared disk, whose outer rim simulates the warm photosphere. Material located outside of the disk's rim is in the ionization shadow and thus represents the neutral zone (Fig. 9).

\subsection{Basic ionization structure}

The results we inferred above imply a significant difference in the ionization structure of the circumbinary environment of AX Per between its quiescent and active phase. The corresponding sketch is shown in Fig. 9. It can be described as follows.

During the quiescent phase, the steady-state STB (Seaquist et al. 1984) model is sufficient to explain the radio emission from symbiotic stars and the wave-like orbitally related variation in the optical LCs (Skopal 2001). However, to explain the shift of light minima to a position preceding the spectroscopic conjunction (Fig. 2, right panel), a more sophisticated model of the density distribution in the binary has to be considered. Hydrodynamical calculations of the structure of stellar winds in symbiotic stars that include effects of the orbital motion suggest a higher density region, twisted from the preceding front of the hot star motion to that of the giant (e.g. Walder 1995; Bisikalo et al. 2006). As a result, the light minima occur before the inferior conjunction of the giant (see also Fig. 13 of Skopal 1998). In Fig. 9 we plotted a scheme of this region as calculated by Bisikalo et al. (2006) for a mass-loss rate from the giant and the hot star of $2 \times 10^{-7}$ and $1.2 \times 10^{-7} M_{\odot} \mathrm{yr}^{-1}$, respectively.

During the active phase, the neutral hydrogen zone has the form of a flared disk encompassing the hot star. Its optically thick rim represents the warm pseudophotosphere. The ionized region fills in the remainder of the space above/below the neutral zone, which is ionized by the hot star. It can be associated 

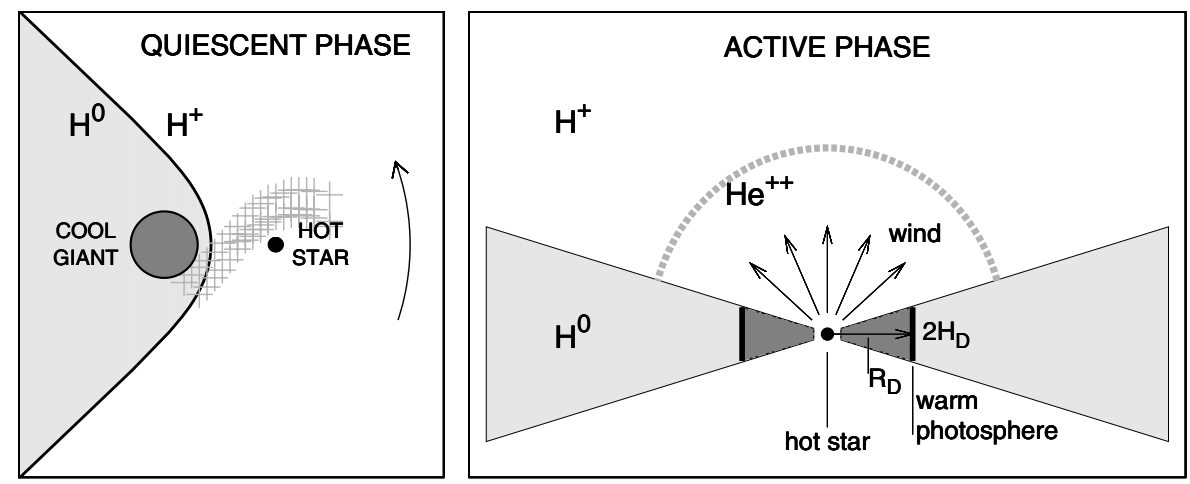

Fig. 9. Sketch of the ionization structure of AX Per during the quiescent phase (left, pole-on view) and around the hot star during the active phase (right, edge-on view). During quiescence, the solid thick line represents the $\mathrm{H}^{0} / \mathrm{H}^{+}$boundary in the steady-state approximation. Including the wind from the hot star and rotation of the binary (denoted by the arrow), a denser region evolves at the preceding front of the hot star, which is further twisted to the front of the giant against its motion (crosses, adapted from Fig. 5 of Bisikalo et al. 2006). The right panel shows a sketch of the ionization structure of the hot component during activity. The neutral zone has the form of a flared disk, whose optically thick rim represents the warm pseudophotosphere. The enhanced wind from the hot star is ionized by its radiation. The $\mathrm{He}^{++}$boundary is denoted by the gray dashed line (see Sect. 4).

with the enhanced stellar wind from the hot star, whose emission is subject to eclipse. The proposed ionization structure is consistent with the UV/near-IR model SED during active phases of symbiotic binaries with high orbital inclination (see Fig. 27 in Skopal 2005) and that of the bipolar stellar wind from the hot star used to model the broad $\mathrm{H} \alpha$ wings (Skopal 2006). The formation mechanism of the neutral disk-like zone is not understood yet. However, first results based on the theory of the wind compression model (Bjorkman \& Cassinelli 1993) support the findings of this paper (Cariková \& Skopal 2011).

\section{Conclusions}

We have investigated a new active phase of the eclipsing symbiotic binary AX Per, which began during 2007. Our main conclusions may be formulated as follows.

(i) AX Per entered a new active phase from 2007.5 (Sect. 3.1.1). After 10 orbital cycles ( $\sim 18.6$ years) we again measured a deep narrow minimum in the LC, which was caused by the eclipse of the hot component by its giant companion. We improved the ephemeris of eclipses (Eq. (1)) and found that their timing is identical (within uncertainties) with that of the inferior conjunction of the giant. We determined the radius of the giant as $R_{\mathrm{g}}=115 \pm 2 R_{\odot}$ and that of the eclipsed object, $R_{\mathrm{e}}=27 \pm 2 R_{\odot}$ (Sect. 3.1.2).

(ii) The new active phase was connected with a significant enhancement of the hot star wind. From quiescence to activity, the mass-loss rate increased from $\sim 9 \times 10^{-8}$ to $\sim 2 \times$ $10^{-6} M_{\odot} \mathrm{yr}^{-1}$, respectively (Sect. 3.2.2, Fig. 6, Table 7). The mean electron concentration within the eclipsed $\mathrm{He}^{++}$zone $\left(R_{\mathrm{HeII}} \sim 50 R_{\odot}\right.$ above the hot star), $\sim 10^{10} \mathrm{~cm}^{-3}$, agrees well with that given independently by the mass loss rate (Sects. 3.2.1 and 3.2.2). The hot star wind creates also a fraction of the $\left[\mathrm{O}_{\mathrm{III}}\right]$ zone. Depending on the mass-loss rate, the nebular lines can be subject to partial eclipse (1994 eclipse) or not (2009 eclipse; Sect. 4.1.1).

(iii) We revealed a significant change of the ionization structure in the binary with respect to the quiescent phase (Sect. 4, Fig. 9). During the 2007-10 active phase we identified a warm stellar source located at the hot star equator (Sect. 3.2.3, Fig. 8). The source had the form of a flared disk, whose outer rim simulated the warm photosphere. It blocks out the ionizing radiation from the hot star, and consequently the material outside of the disk's rim is mostly in neutral form (Sect. 4.2). The ionized region is located above/below the neutral zone and can be associated with the enhanced hot star wind. The formation of the disk-like neutral zone and the increase of the nebular emission during the active phase was connected with the enhanced wind from the hot star (Tables 7 and 8). It is probable that this connection represents a common origin of the warm pseudophotospheres that are observed during the active phases of symbiotic binaries.

Acknowledgements. The authors would like to thank Theodor Pribulla for the acquisition of spectra at the David Dunlap Observatory. We also would like to thank S. Bacci, M. Marinelli, L. Ghirotto and A. Milani for assistance with some of the photometric and spectroscopic observations. We acknowledge with thanks the variable star observations from the AAVSO International Database contributed by observers worldwide and used in this research. The authors are grateful to the referee, Roberto Viotti, for detailed commenting on the manuscript and constructive suggestions. This research was in part supported by a grant of the Slovak Academy of Sciences, VEGA No. 2/0038/10.

\section{References}

Belyakina, T. S. 1979, Izv. Krym. Astrophys. Obs., 59, 133

Belyakina, T. S. 1991, Bull. CrAO, 83, 104

Bisikalo, D. V., Boyarchuk, A. A., Kilpio, E. Yu., Tomov, N. A., \& Tomova, M. T. 2006, Astron. Rep., 50, 722

Bjorkman, J. E., \& Cassinelli, J. P. 1993, ApJ, 409, 429

Brown, R. L., \& Mathews, W. G. 1970, ApJ, 160, 939

Cariková, Z., \& Skopal, A. 2010, New A, 15, 637

Cariková, Z., \& Skopal, A. 2011, in Evolution of Compact Binaries, ed. L. Schmidtobreick, M. R. Schreiber, \& C. Tappert (San Francisco, CA: ASP), ASP Conf. Ser., in press [arXiv: 1106.2420]

Castor, J. I., Abbott, D. C., \& Klein R. I. 1975, ApJ, 195, 157

Corradi, R. L. M., Mikolajewska, J., \& Mahoney, T. J. 2003, Symbiotic Stars Probing Stellar Evolution (San Francisco: ASP), ASP Conf. Ser., 303

Eriksson, M., Johansson, S., \& Wahlgren, G. M. 2004, A\&A, 422, 987

Fekel, F. C., Hinkle, K. H., Joyce, R. R., \& Skrutskie, M. F. 2000, AJ, 120, 3255

Fluks, M. A., Plez, B., The, P. S., et al. 1994, A\&AS, 105, 311

Friedjung, M., \& Viotti, R. F. 1982, The nature of symbiotic stars (Dordrecht: D. Reidel Publishing Co.), IAU Coll., 70

Fujimoto, M. Y. 1982, ApJ, 257, 767

Gurzadyan, G. A. 1997, The Physics and Dynamics of Planetary Nebulae (Berlin: Springer-Verlag)

Henden, A. A., \& Munari, U. 2006, A\&A, 458, 339

Hummer, D. G., \& Storey, P. J. 1987, MNRAS, 224, 801

Iijima, T. 1988, Ap\&SS, 150, 235 
A. Skopal et al.: Formation of a disk structure in the symbiotic binary AX Persei during its $2007-10$ precursor-type activity

Ivison, R. J., Bode, M. F., Evans, A., Skopal, A., \& Meaburn, J. 1993, MNRAS, 264,875

Kenny, H. T., \& Taylor, A. R. 2005, ApJ, 619, 527

Kenyon, S. J., \& Webbink, R. F. 1984, ApJ, 279, 252

Mikolajewska, J., \& Kenyon, S. J. 1992, AJ, 103, 579

Munari, U., \& Zwitter, T. 2002, A\&A, 383, 188

Munari, U., Sordo, R., Castelli, F., \& Zwitter, T. 2005, A\&A, 442, 1127

Munari, U., Siviero, A., Dallaporta, S., et al. 2009a, CBET No. 1757

Munari, U., Siviero, A., Ochner, P., et al. 2009b, PASP, 121, 1070

Munari, U., Siviero, A., Corradi, R. L. M., et al. 2010, CBET No. 2555

Mürset, U., \& Schmid, H. M. 1999, A\&AS, 137, 473

Nussbaumer, H., \& Osterbrock, D. E. 1970, ApJ, 161, 811

Nussbaumer, H., \& Vogel, M. 1987, A\&A, 182, 51

Nussbaumer, H., Schmutz, W., \& Vogel, M. 1995, A\&A, 293, 13

Paczyński, B., \& Rudak, R. 1980, A\&A, 82, 349

Pacźynski, B., \& Żytkow, A. N. 1978, ApJ, 222, 604

Seaquist, E. R., Taylor, A. R., \& Button, S. 1984, ApJ, 284, 202

Siviero, A., Munari, U., Dallaporta, S., et al. 2009, MNRAS, 399, 2139

Skopal, A. 1991, IBVS No. 3603

Skopal, A. 1994, A\&A, 286, 453
Skopal, A. 1998, A\&A, 338, 599

Skopal, A. 2000, CoSka, 30, 21

Skopal, A. 2001, A\&A, 366, 157

Skopal, A. 2005, A\&A, 440, 995

Skopal, A. 2006, A\&A, 457, 1003

Skopal, A. 2007, New A, 12, 597

Skopal, A., Teodorani, M., Errico, L., et al. 2001, A\&A, 367, 199

Skopal, A., Vittone, A. A., Errico, L., et al. 2006, A\&A, 453, 279

Skopal, A., Sekeráš, M., Gonzlez-Riestra, R., \& Viotti, R. F. 2009, A\&A, 507, 1531

Tamura, S., Otsuka, M., Skopal, A., Pribulla, T., \& Vaňko, M. 2003, in Stellar astrophysics - a tribute to Helmut A. Abt, Sixth Pacific Rim Conference, ed. K. S. Cheng, K. C. Leung, \& T. P. Li (Dordrecht: Kluwer Academic Publishers), ASSL, 298, 213

Tutukov, A. V., \& Yangelson, L. R. 1976, Astrofizika, 12, 521

Vogel, M. 1993, A\&A, 274, L21

Walder, R. 1995, in Wolf-Rayet Stars: Binaries, Colliding Winds, Evolution, ed. K. A. van der Hucht, \& P. M. Williams (Dordrecht: Kluwer Academic Publishers), IAU Symp., 163, 420

Wallerstein, G., Willson, L. A., Salzer, J., \& Brugel 1984, A\&A, 133, 137 